\title{
Evolving role of anti-VEGF for diabetic macular oedema: from clinical trials to real life
}

\author{
Tarun Sharma ${ }^{1}$
}

Received: 4 September 2019 / Accepted: 4 September 2019 / Published online: 20 September 2019

(c) The Royal College of Ophthalmologists 2019

\section{Diabetes, a global epidemic}

Diabetes mellitus is a growing global challenge and a major public health issue, particularly due to its microvascular and macrovascular complications [1-4]. Diabetic retinopathy is an important cause of adult-onset blindness and quality of life starts getting affected from the stage of moderate non-proliferative diabetic retinopathy (NPDR) in one eye, an inflexion point; bilateral disease has a greater impact on quality of life than unilateral disease [5]. Therefore, identifying and treating subjects at risk of visual loss is of great importance in preserving vision and preventing complications in this working age population, a key economic driver of society [6].

\section{Anti-VEGF treatment for diabetic macular oedema (DMO)}

Treatment of DMO has undergone a paradigm shift over the last decade. Anti-VEGF therapy has replaced laser photocoagulation as the mainstay of treatment. Encouraging results from the initial anti-VEGF trials-the RISE/RIDE and VIVID/VISTA - led to approval of ranibizumab and aflibercept for the treatment of DMO by the US Food and Drug Administration (FDA) in 2012 and 2014, respectively [7, 8]. Though, off-label bevacizumab has been in use during the period between 2006 and 2012 and thereafter.

Supplementary information The online version of this article (https:// doi.org/10.1038/s41433-019-0590-0) contains supplementary material, which is available to authorized users.

Tarun Sharma

ts3118@cumc.columbia.edu

1 Department of Ophthalmology, Edward S. Harkness Eye Institute, Columbia University College of Physicians and Surgeons, New York, NY, USA
The DRCRnet Protocol T compared the results of three antiVEGF drugs, $2.0 \mathrm{mg}$ aflibercept, $1.25 \mathrm{mg}$ bevacizumab (compounded), or $0.3 \mathrm{mg}$ ranibizumab, in the management of centre-involved DMO. At 2 years, the data showed superiority of aflibercept over bevacizumab and not with ranibizumab (which was seen at 1 year) with regards to mean change in visual acuity (ETDRS letters: 18.3 vs 13.3 vs 16.1 , respectively) in those who had baseline visual acuity of $20 / 50$ or worse. However, if the baseline visual acuity was between 20/32 and 20/40, no difference was observed among these three anti-VEGF drugs [9, 10].

\section{Unanswered question about centre- involving DMO and VA of $\geq 20 / 25$}

What is role of anti-VEGF in the treatment of centreinvolving DMO if the initial or baseline visual acuity was 20/25 or better? Do we need to treat (anti-VEGF or laser) or just observe?

All of the above-mentioned trials (RISE/RIDE/VIVID/ VISTA/Protocol T) had an important eligibility criterion of having baseline visual acuity between 20/32 (or 20/40) and 20/320. In real life situations, many clinicians tend to extrapolate the data from these trials to manage patients who have centre-involving DMO regardless of their initial visual acuity. Therefore, to provide a level 1 evidence, DRCR.net conducted a randomized clinical trial (protocol V) comparing aflibercept with laser and observation in patients with DMO that involved the centre of the macula and had a visual acuity of $20 / 25$ or better and published results in the April 2019 issue JAMA [11].

\section{Protocol V}

The protocol $\mathrm{V}$ concluded that at 2 years follow-up, no difference was observed-with regard to the primary outcome defined as visual acuity loss of $\geq 5$ ETDRS letters-among 
eyes that were managed with aflibercept, laser photocoagulation, or observation [11]. Eyes randomized to aflibercept group received monthly injection if there was either an improvement or deterioration in visual acuity and/or central retinal thickness: change in $\geq 5$ letters or $\geq 10 \%$ of thickness during the last two visits. However, it is critical to know that eyes that were randomized to laser photocoagulation or observation group were allowed to cross-over to aflibercept group if there was a deterioration in visual acuity from baseline by either $\geq 2$ lines (at least 10 letters) at any visit or by 1-2 lines (5-9 letters) at two consecutive visits. So, when we look at the median number of injections over 2 years, they were almost the same in all the three groups: eight in aflibercept, seven in laser, and nine in the observations group. Therefore, in the end, it is not a comparison of monotherapy (aflibercept vs laser vs observation, a pure homogenous group in each category). About 25\% (cumulative probability, 26\%) of the laser group and 34\% (cumulative probability, 36\%) of the observation group received aflibercept during the study period. So, both laser and observation groups had two subsets of participants: one is the original randomized group, and the second with aflibercept. Were there any differences in the outcome between two subsets within the group or between groups, maybe subsequent secondary analysis might answer that?

The protocol $\mathrm{V}$ also evaluated effect on diabetic retinopathy severity scale (Supplement 3, eTable 7) and observed that two-step worsening was significantly less in the aflibercept group compared with laser photocoagulation and observation groups $(4 \%, 10 \%$, and $11 \%$, respectively). Since this was an exploratory outcome, this needs to be reviewed in future studies. Of interest are the results from the PANORAMA study, a randomized trial that investigated if anti-VEGF therapy (aflibercept vs sham) could prevent PDR or centre-involving DMO (CI-DMO) in eyes with moderately severe or severe NPDR; the 1-year data showed that around $40 \%$ in sham group developed visionthreatening changes (proliferative diabetic retinopathy or CI-DMO) than just $10 \%$ who received aflibercept. So, the role of anti-VEGF continues to evolve in terms of treatment of diabetic retinopathy as well as prevention of its progression to advanced disease [12].

\section{A comprehensive care for DMO in real life}

To treat or just observe DMO, a vision threatening complication which may occur at any stage of diabetic retinopathy, is an important question a clinician needs to address [13]. The Early Treatment Diabetic Retinopathy Study (ETDRS) showed that about $40 \%$ of eyes with macular oedema had visual acuity of $\geq 20 / 20$ at the baseline [14]. The protocol $\mathrm{V}$ demonstrated that observation alone is one of the options, as nearly two-thirds of the eye in this group did not experience loss of vision of even one line over 2-year period. However, the relationship between DMO with relatively good vision and quality of life has not been investigated in any of these pivotal clinical trials. In real life, many factors that influence the clinician's decision to treat or observe DMO are: compliance to follow-up, speed of worsening of diabetic retinopathy, status of the fellow eye, cost of the drug, risk of intravitreal injection, patient's fears and expectations, and co-morbid diseases like hypertension, dyslipidemia, renal status.

Diabetic retinopathy is a disease on continuum. Besides making diagnosis and treating it, we all need to educate our patients the benefit of keeping good glycaemic control and screening them early for retinopathy.

\section{Compliance with ethical standards}

Conflict of interest The author declares that he has no conflict of interest.

Publisher's note Springer Nature remains neutral with regard to jurisdictional claims in published maps and institutional affiliations.

\section{References}

1. Zimmet PZ. Diabetes and its drivers: the largest epidemic in human history? Clin Diabetes Endocrinol. 2017;3:1. https://doi. org/10.1186/s40842-016-0039-3.

2. Shi Y, Vanhoutte PM. Macro- and microvascular endothelial dysfunction in diabetes. J Diabetes. 2017;9:434-49.

3. Rangel EB, Rodrigues CO, de Sa JR. Micro- and macrovascular complications in diabetes mellitus: preclinical and clinical studies. J Diabetes Res. 2019;2019:2161085. https://doi.org/10.1155/ 2019/2161085.

4. Gedebjerg A, Almdal TP, Berencsi K, Rungby J, Nielsen JS, Witte DR, et al. Prevalence of micro- and macrovascular diabetes complications at time of type 2 diabetes diagnosis and associated clinical characteristics: a cross-sectional baseline study of 6958 patients in the Danish DD2 cohort. J Diabetes Complications. 2018;32:34-40. https://doi.org/10.1016/j.jdiacomp.2017.09.010.

5. Mazhar K, Varma R, Choudhury F, McKean-Cowdin R, Shtir CJ, Azen SP. Severity of diabetic retinopathy and health-related quality of life: Los Angeles Latino Eye Study Group. Ophthalmology. 2011;118:649-55.

6. Tan GS, Cheung N, Simo R, Cheung GC, Wong TY. Diabetic macular edema. Lancet Diabetes Endocrinol. 2017;5:143-55.

7. Brown DM, Nguyen QD, Marcus DM, Boyer DS, Patel S, Feiner L, et al. Long-term outcomes of Ranibizumab therapy for diabetic macular edema: the 36-months results from two phase III trials RISE and RIDE. Ophthalmology. 2013;120:2013-22.

8. Heier JS, Korobelnik JK, Brown DM, Schmidt-Erfurth U, Do DV, Midena E, et al. Intravitreal Aflibercept for diabetic macular edema: 148-week results from the VISTA and VIVID studies. Ophthalmology. 2016;123:2376-85.

9. Wells JA, Glassman AR, Ayala AR, Jampol LM, Bressler NM, Bressler SB, et al. Aflibercept, Bevacizumab, or Ranibizumab for 
diabetic macular edema: two-year results from a comparative effectiveness randomized clinical trial. Ophthalmology. 2016;123: 1351-9.

10. Ross EL, Hutton DW, Stein JD, Bressler NM, Jampol LM, Glassman AR. Diabetic Retinopathy Clinical Research Network. Cost-effectiveness of Aflibercept, Bevacizumab, and Ranibizumab for Diabetic Macular Edema Treatment: analysis from the diabetic retinopathy clinical research network comparative effectiveness trial. JAMA Ophthalmol. 2016;134:888-96.

11. Baker CW, Glassman AR, Beaulieu WT, Antoszyk AN, Browning DJ, Chalam KV, et al. Effect of initial management with aflibercept vs laser photocoagulation vs observation on vision loss among patients with diabetic macular edema involving the center of the macula and good visual acuity a randomized clinical trial. JAMA. 2019;321:1880-94.

12. Wykoff CC. Intravitreal aflibercept for moderately severe to severe non-proliferative diabetic retinopathy (NPDR): the phase 3 PANORAMA study. Presented at: Angiogenesis, exudation and Degeneration 2019; February 9, 2019; Miami, FL.

13. Yau JW, Rogers SL, Kawasaki R, Lamoureux EL, Kowalski JW, Bek T, et al. Global prevalence and major risk factors of diabetic retinopathy. Diabetes Care. 2012;35:556-64.

14. Early Treatment Diabetic Retinopathy Study Research Group. Early Treatment Diabetic Retinopathy Study design and baseline patient characteristics: ETDRS report number 7. Ophthalmology. 1991;98:741-6. 